\title{
Meiotic behavior and pollen viability in Moringa oleifera (Moringaceae) cultivated in southern Brazil
}

\author{
N. Silva ${ }^{1}$, A.B. Mendes-Bonato ${ }^{1}$, J.G.C. Sales ${ }^{2}$ and M.S. Pagliarini ${ }^{1}$ \\ ${ }^{1}$ Departamento de Biologia Celular e Genética, \\ Universidade Estadual de Maringá, Maringá, PR, Brasil \\ ${ }^{2}$ Departamento de Agronomia, Universidade Estadual de Maringá, \\ Maringá, PR, Brasil
}

Correspondence author: M.S. Pagliarini

E-mail:mspagliarini@uem.br

Genet. Mol. Res. 10 (3): 1728-1732 (2011)

Received May 18, 2011

Accepted August 8, 2011

Published August 18, 2011

DOI http://dx.doi.org/10.4238/vol10-3gmr1490

\begin{abstract}
Although originally from India, Moringa oleifera is now cultivated throughout most of the tropics, including Brazil. Despite its multipurpose value for food and traditional medicine, little is known about the meiotic behavior and pollen viability of M. oleifera. We evaluated microsporogenesis and pollen viability in eleven plants grown in southern Brazil (Maringá, Paraná). Bud flowers were collected in different stages of development. All plants that we analyzed presented $2 \mathrm{n}=28$ chromosomes, as previously reported for this species. Chromosomes paired as bivalents. Meiotic abnormalities were rare and metaphase I was the most affected phase. Pollen viability was superior to $88 \%$. Tripolar spindles in metaphase II, leading to the formation of unreduced gametes, were
\end{abstract}


recorded in some plants at a low frequency.

Key words: Moringa oleifera; Meiosis; Tripolar spindles; Unreduced gametes; Pollen viability

\section{INTRODUCTION}

Moringa oleifera Lam. belongs to a monogeneric family of shrubs and trees, the Moringaceae (Ramachandran et al., 1980; Muluvi et al., 1999). It is native to the sub-Himalayan tracts where it is known by several regional names (Jahn et al., 1986; Kantharajah and Dodd, 1991; Fahey, 2005). Many reports have described its nutritional and medicinal properties, transforming it into a panacea (Fahey, 2005). Muluvi et al. (1999) and Fahey (2005) enumerated its several uses with great propriety.

Although utilized by people in ancient times, $M$. oleifera is now widely cultivated and has become naturalized in many locations in the tropics (Fahey, 2005). It is an important crop in Brazil, mainly in tropical and undeveloped regions, where seeds are used as a natural coagulant for water purification. In temperate southern Brazil, this species is being evaluated for oil production. Detailed studies of genetic variability are limited (Ramachandran et al., 1980; Muluvi et al., 1999), but they indicate considerable potential for improvement. $M$. oleifera plants are being cultivated at the farm of the State University of Maringá to evaluate oil seed production. At this time, enormous genetic variability in morphology has been found among trees. Considering that seed production is dependent on viable gametes, the aim of this research was to evaluate the meiotic behavior of some plants.

\section{MATERIAL AND METHODS}

A total of $11 \mathrm{M}$. oleifera plants from the Experimental Farm of the State University of Maringá (Maringá, Paraná, Brazil) were analyzed for their meiotic behavior and pollen viability.

For meiotic studies, flower buds were collected and fixed in Carnoy ( 3 alcohol:1 acetic acid) for $24 \mathrm{~h}$, and then stored under refrigeration in $70 \%$ ethanol. Meiocytes were obtained by squashing anthers in a drop of $1 \%$ propionic carmine. All meiotic phases were evaluated under light microscopy and meiotic abnormalities scored.

Pollen viability was determined by differential staining of viable and nonviable pollen grains with $1 \%$ propionic carmine. A total of 500 pollen grains were analyzed per plant. Pollen fertility was evaluated after $30 \mathrm{~min}$ of suspension in a drop of staining. Microphotographs were taken using a Wild Leitz microscope with Kodak Imagelink - HQ, ISO 25 black and white film.

\section{RESULTS}

Cytological analyses of meiocytes in prophase I revealed that chromosomes are very small (Figure 1a-c) with several heterochromatic regions of different sizes along the bivalents in pachytene (Figure 1a,b). A large heterochromatic block is found in the nucleolar organizer region. Fourteen small bivalents were visualized at diakinesis (Figure 1c), sometimes with a considerable distance between homologous chromosomes. Metaphase I (Figure 1d) was the most abnormal phase in the meiotic process. Precocious chromosome migration to the poles 
was found in several meiocytes (Table 1). Laggards in anaphase I (Figure 1e,f) were detected in some plants. Both abnormalities gave rise to micronuclei in telophase (Figure 1g). In the second division, the abnormalities were also characterized by precocious chromosome migration to the poles in metaphase II (Figure 1h), laggards in anaphase II (Figure 1i), and micronuclei in telophase II (Figure 1j). In the second division, tripolar spindles were recorded in metaphase II (Figure 2a), leading to trinucleated telophase II (Figure 2b,c). In some meiocytes, the convergence of spindles led to the formation of a restitutional nucleus (Figure 1c). Such telophases generated triads with two $n$ microspores and one unreduced microspore (Figure $1 \mathrm{e}$ ). The products were two $n$ pollen grains and one unreduced pollen grain (Figure $2 \mathrm{f}$ ). When the convergence of spindles was not total, a triad with a binucleated microspore was formed (Figure 1d). Pollen viability was over $88 \%$. Well-developed pollen grains showed three apertures (Figure 1k). A sterile pollen grain is shown in Figure 11.

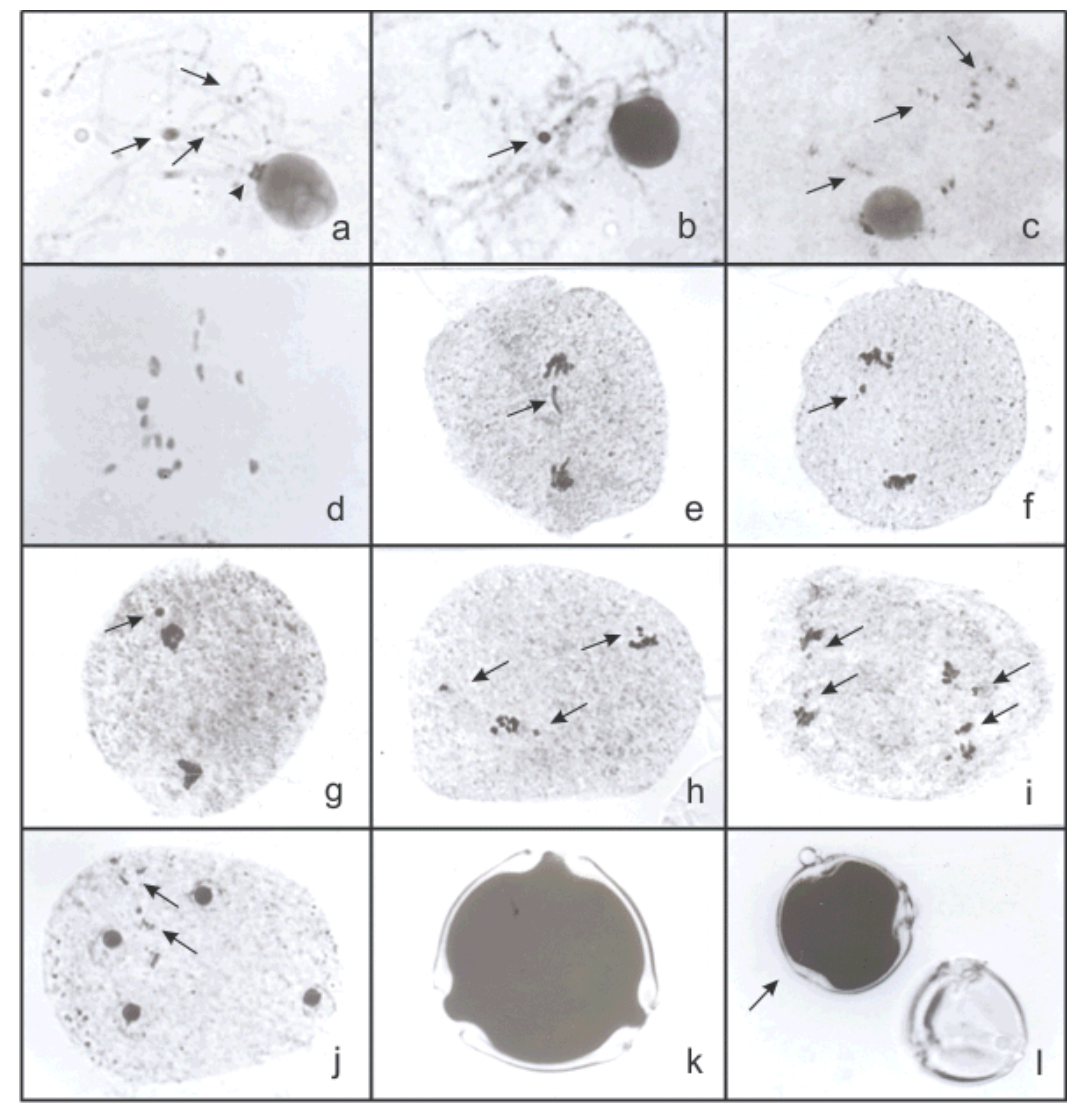

Figure 1. Aspects of microsporogenesis in Moringa oleifera. a.b. Meiocytes in pachytene showing small chromosomes with several heterochromatic regions of different sizes (arrows). Observe the heterochromatic block at the nucleolus organizer region (arrowhead). c. Diakinesis with small bivalents. Observe the distance between homologous chromosomes in some bivalents (arrows). d. Early metaphase I with bivalents not yet congregated. e.f. Anaphase I with laggards (arrow). g. Telophase I with a micronucleus (arrow). h. Metaphase II with precocious chromosome migration to the poles (arrows). i. Anaphase II with laggards (arrows). j. Telophase II with micronuclei (arrows). k. Fertile pollen grain with three apertures. l. Fertile (arrow) and sterile pollen grains. 


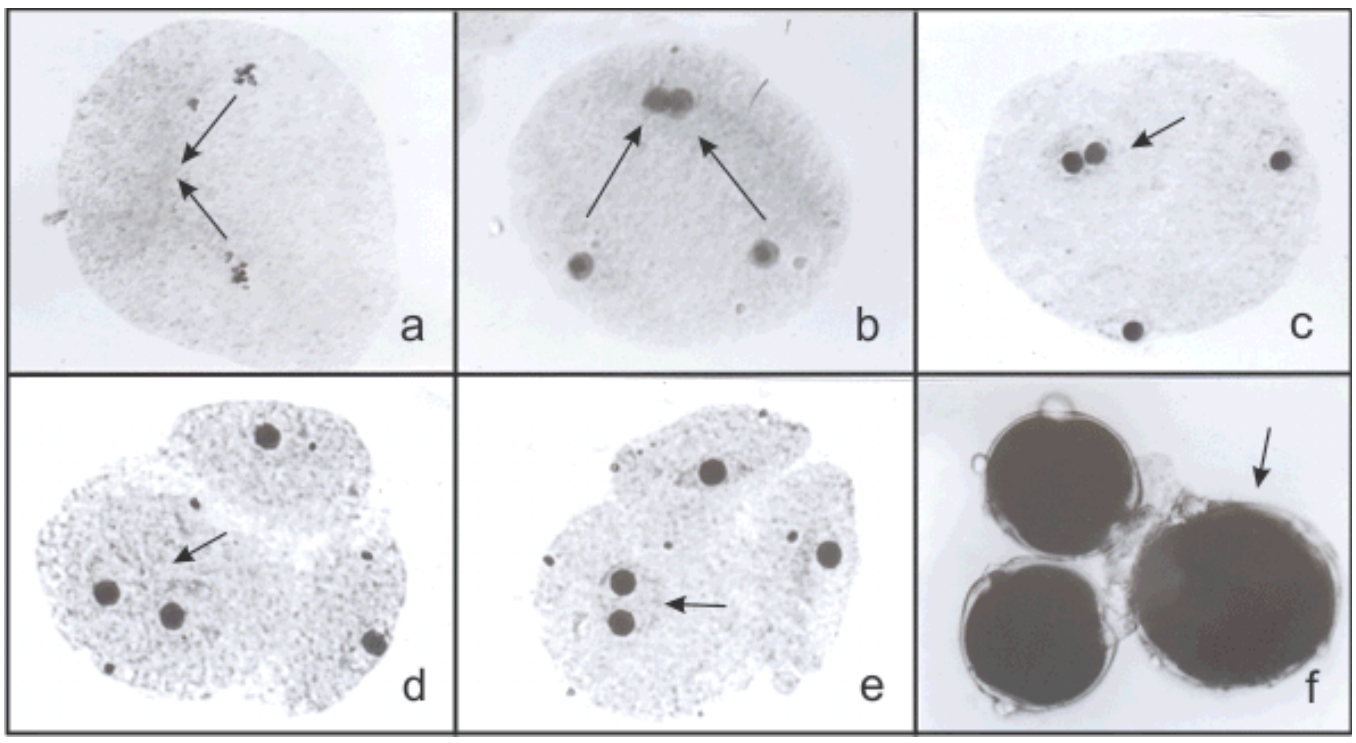

Figure 2. Tripolar spindle formation. a. Metaphase II with tripolar spindles. Observe the convergence of spindles to one pole (arrows). b. Telophase II with tripolar spindles, but with non-restitutional nucleus (arrows). c. Telophase II with tripolar spindles and the formation of a restitutional nucleus (arrow). d. Triad with a binucleated microspore (arrow). e. Triad with an unreduced microspore (arrow). f. Unreduced pollen grain (arrow) and two $n$ pollen grains.

Table 1. Number of cells analyzed/number of abnormal cells per plant of Moringa oleifera and pollen viability.

\begin{tabular}{|c|c|c|c|c|c|c|c|c|c|c|c|}
\hline \multirow[t]{2}{*}{ Plant } & \multicolumn{9}{|c|}{ Meiotic phases } & \multirow[t]{2}{*}{ No. of cells } & \multirow[t]{2}{*}{ Pollen viability (\%) } \\
\hline & Diakinesis & MI & $\mathrm{AI}$ & TI & PII & MII & AII & TII & Tetrad & & \\
\hline 1 & $197 / 0$ & $28 / 6$ & $7 / 0$ & $71 / 0$ & - & $22 / 2$ & $2 / 0$ & $283 / 31$ & $137 / 4$ & $747 / 43$ & 94.8 \\
\hline 2 & $160 / 0$ & $42 / 0$ & - & $93 / 2$ & $42 / 0$ & $80 / 0$ & $1 / 0$ & $125 / 0$ & $125 / 0$ & $668 / 2$ & 95.8 \\
\hline 3 & $53 / 0$ & $62 / 3$ & $11 / 1$ & $107 / 7$ & $55 / 0$ & $54 / 0$ & $2 / 0$ & $85 / 0$ & $117 / 0$ & $546 / 11$ & 89.4 \\
\hline 4 & $97 / 0$ & $57 / 11$ & $6 / 0$ & $55 / 0$ & $28 / 0$ & $42 / 0$ & $6 / 0$ & $90 / 0$ & $95 / 0$ & $476 / 11$ & 95.2 \\
\hline 5 & $130 / 0$ & $87 / 32$ & $52 / 3$ & $127 / 17$ & $36 / 0$ & $41 / 0$ & $12 / 0$ & $132 / 3$ & $200 / 0$ & $817 / 55$ & 92.2 \\
\hline 6 & $134 / 0$ & $273 / 28$ & $28 / 2$ & $202 / 3$ & $37 / 0$ & $42 / 0$ & $2 / 0$ & $231 / 3$ & $150 / 0$ & $1099 / 36$ & 96.2 \\
\hline 7 & $77 / 0$ & $42 / 3$ & $10 / 0$ & $60 / 0$ & $11 / 0$ & $28 / 0$ & $6 / 0$ & $83 / 0$ & $100 / 0$ & $417 / 3$ & 91.8 \\
\hline 8 & $22 / 0$ & $46 / 0$ & $12 / 0$ & $102 / 0$ & $32 / 0$ & $46 / 7$ & $3 / 0$ & $56 / 11$ & $120 / 0$ & $439 / 18$ & 89.4 \\
\hline 9 & $135 / 0$ & $56 / 0$ & $80 / 0$ & $18 / 0$ & $100 /-$ & $45 / 12$ & $2 / 0$ & $140 / 6$ & $105 / 0$ & $681 / 18$ & 88.0 \\
\hline 10 & $97 / 0$ & $84 / 22$ & $22 / 7$ & $91 / 19$ & $47 / 4$ & $62 / 0$ & $3 / 2$ & $128 / 30$ & $100 / 0$ & $634 / 84$ & 89.8 \\
\hline 11 & $92 / 0$ & $100 / 18$ & $12 / 0$ & $40 / 6$ & $6 / 0$ & $58 / 7$ & $3 / 1$ & $220 / 20$ & $50 / 0$ & $581 / 52$ & 89.8 \\
\hline Total & $1194 / 0$ & $877 / 123$ & $240 / 13$ & $966 / 54$ & $394 / 4$ & $520 / 28$ & $42 / 3$ & $1573 / 104$ & $1299 / 4$ & & \\
\hline
\end{tabular}

MI = metaphase I; AI = anaphase I; TI = telophase I; PII = prophase II; MII = metaphase II; AII = anaphase II; $\mathrm{TII}=$ telophase II.

\section{DISCUSSION}

The chromosome number recorded in the plants analyzed, $2 \mathrm{n}=28$, was previously reported by Mendioro et al. (2004). The meiotic behavior at prophase I in bivalents confirms the diploid condition of this species. The distance between homologues in the bivalents predisposes them to precocious chiasma terminalization, leading to precocious chromosome migration of univalents to the poles in metaphase I. Laggard chromosomes in anaphase I and micronuclei 
in telophase I were found in low frequency, showing that the chromosomes with irregular segregation were incorporated in the telophase nuclei. This meiotic behavior is common in higher plants (Pagliarini, 2000). In the second division, abnormalities decreased as also reported in the plants analyzed by Mendioro et al. (2004). The number of tetrads with micronuclei was very low, and pollen viability was high. Moringa oleifera is an outcrossing species (Ramachandran et al., 1980; Jyothi et al., 1990) and needs a lot of viable pollen to guarantee seed formation. Pollen viability was high and similar to that reported by Mendioro et al. (2004).

The occurrence of a tripolar spindle found in several plants, although in low frequency, is facilitated in dicotyledonous species, where cytokinesis is simultaneous, occurring after telophase II (Harlan and deWet, 1975; Veilleux, 1985; Bretagnolle and Thompson, 1995). The product of this abnormal spindle orientation, a triad, consists of a binucleated or an unreduced microspore and two $n$ microspores. Until now, there has been no report of such abnormality in $M$. oleifera. Unreduced gametes are considered to be the main source of polyploidy and are widespread among plants (Harlan and deWet, 1975; Veilleux, 1985; Bretagnolle and Thompson, 1995). Several mechanisms, including tripolar spindles, have been reported to cause meiotic nuclear restitution (Ramanna, 1979). The number of meiocytes with tripolar spindles in metaphase II and triads was very small. Bretagnolle and Thompson (1995) showed that the frequency of unreduced gametes varies among individuals within plants and also among flowers of an individual plant. But, at any rate, unreduced gametes may contribute to the evolutionary history of a taxonomic group by the creation of polyploids. Although not yet reported in $M$. oleifera, polyploidy could be very interesting to better exploit the nutritional and the medicinal properties of this species.

\section{REFERENCES}

Bretagnolle F and Thompson JD (1995). Gametes with the somatic chromosome number: mechanisms of their formation and role in the evolution of autopolyploid plants. New Phytol. 129: 1-22.

Fahey JW (2005). Moringa oleifera: a review of the medicinal evidence for its nutritional, therapeutic, and prophylactic properties. Part 1. Trees Life J. 1: 5-23.

Harlan JR and deWet JMJ (1975). On Ö. Winge and a prayer: the origins of polyploidy. Bot. Rev. 41: 361-390.

Jahn SAA, Musnad HA and Burgstaller H (1986). The tree that purifies water: cultivating multipurpose Moringaceae in the Sudan. Unasylva 152: 23-28.

Jyothi PV, Atluri JB and Reddi CS (1990). Pollination ecology of Moringa oleifera (Moringaceae). Proceed. Ind. Acad. Sci. (Plant Sci.) 100: 33-42.

Kantharajah AS and Dodd WA (1991). Rapid clonal propagation of M. oleifera Lam., using tissue culture. South Ind. Hort. 39:224-228.

Mendioro MS, Diaz MGQ, Alcantara MTB, Hilario OJ, et al. (2004). Cytological studies of selected medicinal plants: Euphorbia pulcherrima Willd. ex Klotz., Moringa olieferu Lam., Catharanthus roseus (L.) Don. and Chrysantthemum indicum Linn. Philipp. J. Sci. 134: 31-38.

Muluvi GM, Sprent JI, Soranzo N, Provan J, et al. (1999). Amplified fragment length polymorphism (AFLP) analysis of genetic variation in Moringa oleifera Lam. Mol. Ecol. 8: 463-470.

Pagliarini MS (2000). Meiotic behavior of economically important plant species: the relationship between fertility and male sterility. Genet. Mol. Biol. 23: 997-1002.

Ramachandran C, Peter KV and Gopalakrishnan PK (1980). Drumstick (Moringa oleifera): a multipurpose Indian vegetable. Econ. Bot. 34: 276-283.

Ramanna MS (1979). A re-examination of the mechanisms of $2 \mathrm{n}$ gamete formation in potato and its implications for breeding. Euphytica 28: 537-561.

Veilleux R (1985). Diploid and polyploid gametes in crop plants: mechanisms of formation and utilization in plant breeding. Plant Breed. Rev. 3: 253-288. 